\title{
ESTUDO SOBRE O ESCOAMENTO GRAVITACIONAL DE PÓS DE ALUMINA NA DESCARGA DE RESERVATÓRIOS
}

\section{M. SOUSA e M. C. FERREIRA}

\author{
Universidade Federal de São Carlos, Departamento de Engenharia Química \\ E-mail para contato: mariaf@ufscar.br
}

\begin{abstract}
RESUMO - O padrão de escoamento e as vazões de descarga de pós em silos dependem das características físico-químicas dos materiais, das dimensões e geometrias dos reservatórios e da carga e compactação do pó. Dada a complexidade dos fenômenos envolvidos, a fundamentação teórica para a previsão das condições de escoamento é complexa e ainda pouco abordada em cursos de graduação em engenharia. Este trabalho teve como objetivo avaliar a descarga de pós de alumina em reservatórios cilíndricos de base cônica, construídos em ferro galvanizado. Variou-se o diâmetro de saída do reservatório, o ângulo de inclinação da base e a distribuição granulométrica das partículas. Os pós foram caracterizados quanto à distribuição granulométrica, morfologia, ângulo de repouso e densidades bulk solta e compactada. Foi realizado um planejamento fatorial $2^{3}$, obtendo-se uma equação empírica para a estimativa da vazão mássica em função das variáveis investigadas.
\end{abstract}

\section{INTRODUÇÃO}

A descarga a partir de reservatórios é uma das operações unitárias mais comuns envolvendo o escoamento de materiais particulados, estando presente em diversos setores industriais: cerâmico, farmacêutico, alimentício, etc. Segundo Gomide (1983), a utilização de tanques, depósitos e silos para a armazenagem de produtos é indispensável nas indústrias de processos químicos, pois eles operam como "pulmões" normalizadores de vazão dos produtos intermediários entre as unidades do processo.

Apesar da ampla aplicabilidade industrial, prever o escoamento de pós é uma tarefa complexa e, quando comparado ao escoamento de gases e líquidos, é um tópico ainda pouco estudado em cursos de graduação em Engenharia. Em geral, o escoamento de um fluido pode ser descrito a partir de equações de conservação de massa e momento, contudo no caso dos sólidos, as características do escoamento dependem fortemente das propriedades do material particulado, das interações com a parede dos recipientes, da forma como o material é empacotado, etc. (Woodcock e Mason, 1987).

Em vista da dificuldade de se desenvolver modelos teóricos para a previsão do escoamento e com o objetivo de entender os fenômenos envolvidos nesta operação, muitos autores desenvolveram equações empíricas para estimativa da vazão de descarga de pós em função de variáveis estruturais dos reservatórios e dos materiais particulados. A equação de Beverloo (1961) (appud Verghese e Nedderman, 1995), ajustada para a descarga de materiais granulares de escoamento livre a partir de orifícios circulares em reservatórios com 
base plana, é uma das precursoras. Nesta equação, a vazão mássica de descarga (W) é expressa em função da densidade do material nas condições de escoamento ( $\rho$ ), da aceleração da gravidade ( $\mathrm{g}$ ) do diâmetro do orifício de saída do silo (D) e do diâmetro das partículas $\left(\mathrm{d}_{\mathrm{p}}\right)$. Os parâmetros $\mathrm{C}$ e $\mathrm{k}$ dependem da natureza do material particulado, assumindo normalmente os valores de 0,58 e 1,5. Esta equação é dada por:

$$
W=C \rho g^{\frac{1}{2}}\left(D-k d_{p}\right)^{5 / 2}
$$

De acordo com Verghese e Nedderman (1995), a Equação 1 descreve satisfatoriamente a vazão de descarga para materiais não-coesivos com diâmetros de partículas maiores que $500 \mu \mathrm{m}$. Abaixo desse diâmetro, a vazão é menor do que a prevista pela correlação, decrescendo rapidamente com a diminuição do diâmetro das partículas. Para materiais com tamanhos menores do que $600 \mu \mathrm{m}$, os autores sugeriram a correção do fator $\mathrm{C}$, sendo $\alpha$ o semi-ângulo de inclinação do silo, medido em relação ao eixo vertical.

$$
C^{*}=0,5 \tan (\alpha)^{-0,35}\left(1-1,46 \times \frac{10^{-8}}{d_{p}^{2}}\right)^{\frac{1}{2}} \text { se } C^{*}>0,5 . \text { Senão } C^{*}=0,5
$$

O presente trabalho teve como objetivo avaliar a descarga gravitacional de pós de alumina a partir de reservatórios cilíndricos e analisar a influência de características estruturais do reservatório (inclinação da base cônica e diâmetro do orifício de saída) e do material particulado (diâmetro médio dos pós) sobre essa vazão de descarga. Os pós foram caracterizados através da determinação das densidades bulk aerada e consolidada, massas específicas, ângulo de repouso e índices de Hausner. A partir de uma análise estatística, efetuada a partir de um planejamento fatorial $2^{3}$ foi ajustada uma equação empírica para a estimativa das vazões de descarga e os resultados foram comparados também com estimativas de correlações da literatura.

\section{METODOLOGIA}

Os pós de alumina, com duas diferentes distribuições granulométricas (ALR100 e ALR180) foram adquiridos da ELUMA (São Paulo-SP). As distribuições granulométricas foram obtidas utilizando o equipamento Malvern Mastersizer 2000, com dispersão em via úmida, em triplicata. A partir das distribuições, foram obtidos os diâmetros médio de peneira $\left(d_{50}\right)$ e de Sauter $\left(d_{S}\right)$. A massa específica das partículas $\left(\rho_{\mathrm{p}}\right)$ foi determinada por picnometria líquida com água, em duplicata. A morfologia foi avaliada através de microscopia eletrônica de varredura, utilizando o MEV Inspect S50. As densidades bulk aerada e consolidada foram obtidas segundo a técnica descrita por Campos e Ferreira (2013), na qual uma proveta de $250 \mathrm{ml}$, com $22 \mathrm{~mm}$ de diâmetro, era inserida em um dispositivo de madeira com uma trava localizada a $3 \mathrm{~cm}$ da base da proveta. O pó era inserido na proveta através de um funil. Para a compactação, a proveta era levantada até a altura da trava e posteriormente solta, repetidamente e manualmente. Estes ensaios foram realizados 4 vezes para cada material e os valores médios foram utilizados. Os índices de Hausner (IH) foram estimados calculando- 
se a razão entre densidade bulk consolidada e aerada. O ângulo de repouso (AR) foi medido segundo a técnica descrita por Woodcock e Mason (1987), na qual o pó era despejado em uma placa de acrílico lisa por meio de um funil posicionado a $10 \mathrm{~cm}$ de altura. Esses ensaios foram repetidos 10 vezes para cada pó.

Foram construídos dois reservatórios cilíndricos com base cônica, em ferro galvanizado, com $10 \mathrm{~cm}$ de diâmetro e diferentes ângulos de inclinação da base cônica (cone interno com semi-ângulos de $15^{\circ}$ e $45^{\circ}$ ) e com a possibilidade de alteração dos diâmetros dos orifícios de saída $(15 \mathrm{~mm}$ e $25 \mathrm{~mm})$ na parte inferior do reservatório, por meio do rosqueamento das partes móveis. As peças intercambiáveis foram confeccionadas de forma a manter inalterada a angulação do cone em sua parede interna. Os reservatórios comportam volumes aproximados de $3300 \mathrm{~cm}^{3}$ e $2720 \mathrm{~cm}^{3}$. A vazão mássica de descarga dos pós nos reservatórios foi medida, em triplicata, com o auxílio de um cronômetro e de uma balança eletrônica 9094 (Toledo, Brasil) posicionada imediatamente abaixo da saída do silo. A balança dispunha de uma placa de aquisição de dados, a qual era conectada em um computador, sendo registrados os dados de massa em função do tempo. Para cada ensaio, colocou-se cerca de $3 \mathrm{~kg}$ de pó no silo no momento que o ensaio seria realizado, com o auxílio de um funil. Os ensaios foram efetuados segundo um planejamento experimental $2^{3}$, elaborado usando o software Minitab 17, tendo a vazão mássica de descarga como variável resposta. Os fatores de análise foram o diâmetro de peneira dos pós, o diâmetro do orifício de saída do reservatório e o semi-ângulo de inclinação do silo.

\section{RESULTADOS E DISCUSSÕES}

A Figura 1 apresenta as curvas de distribuição granulométrica para os pós, nas quais estão representadas as médias das réplicas. Pelas curvas, percebe-se que o pó denominado ALR180 possui maior fração de finos em comparação ao ALR100. O diâmetro de peneira $\left(\mathrm{d}_{50}\right)$ foi obtido fixando-se o volume de $50 \%$ e o de Sauter $\left(\mathrm{d}_{\mathrm{S}}\right)$ foi fornecido pelo próprio software do Malvern. Os valores obtidos são mostrados na Tabela 1.

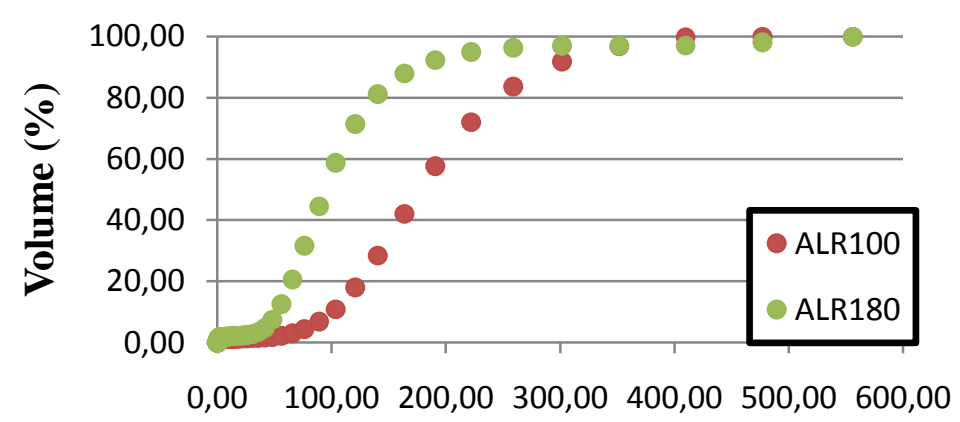

Diâmetro da partícula $(\mu \mathrm{m})$

Figura 1 - Distribuições granulométricas dos pós. 
As microscopias eletrônicas, mostradas nas Figuras 2(a) e 2(b) mostram que os pós de alumina são formados por partículas de formato irregular que não formam aglomerados.
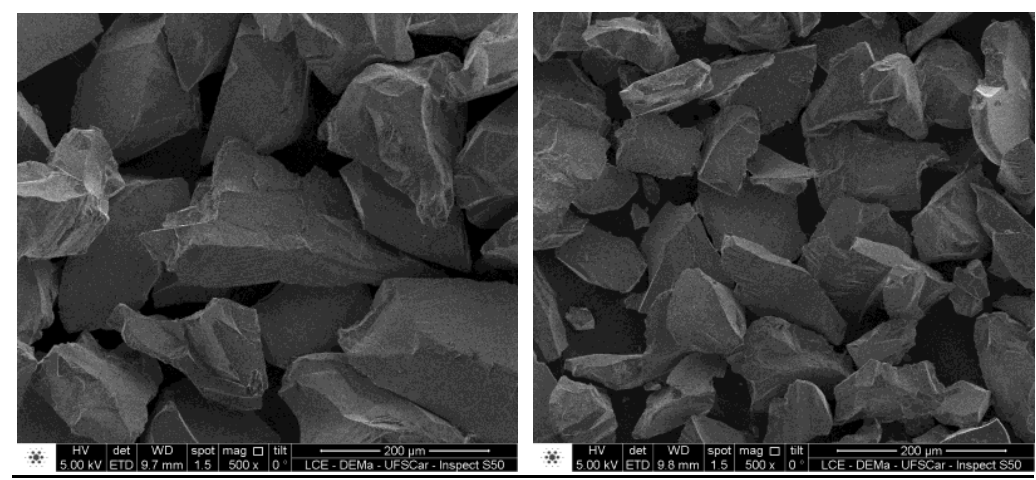

Figura 2 - Microscopias eletrônica para (a) ALR100; (b) ALR180.

Além dos diâmetros, na Tabela 1 são apresentados os valores das massas específicas das partículas, densidades bulk areada e compactada, ângulos de repouso e índices de Hausner. Nota-se que os valores de $\rho_{\mathrm{p}}$ foram similares para ambas as granulometrias analisadas e compatíveis com o valor de $3,94 \mathrm{~g} / \mathrm{cm}^{3}$, citado na ficha técnica do fabricante (Elfusa, São Paulo).

Tabela 1 - Propriedades dos materiais particulados

\begin{tabular}{|c|c|c|c|c|c|c|c|}
\hline Material & $\mathrm{d}_{50}(\mu \mathrm{m})$ & $\mathrm{d}_{\mathrm{S}}(\mu \mathrm{m})$ & $\rho_{\mathrm{p}}\left(\mathrm{g} / \mathrm{cm}^{3}\right)$ & $\rho_{\mathrm{a}}\left(\mathrm{g} / \mathrm{cm}^{3}\right)$ & $\rho_{\mathrm{c}}\left(\mathrm{g} / \mathrm{cm}^{3}\right)$ & AR $\left(^{\circ}\right)$ & $\mathrm{IH}$ \\
\hline ALR100 & $177,2 \pm 0,8$ & $42,02 \pm 0,03$ & $3,91 \pm 0,03$ & $1,54 \pm 0,01$ & $1,92 \pm 0,01$ & $25 \pm 1$ & $1,23 \pm 0,01$ \\
\hline ALR180 & $94,3 \pm 0,3$ & $23,5 \pm 0,1$ & $3,80 \pm 0,04$ & $1,68 \pm 0,01$ & $2,08 \pm 0,02$ & $26 \pm 2$ & $1,24 \pm 0,01$ \\
\hline
\end{tabular}

O índice de Hausner e o ângulo de repouso são obtidos a partir de medidas simples e utilizados para caracterizar a escoabilidade de um material particulado. Em geral, é esperado que a coesividade de um pó diminua com o aumento do tamanho das partículas (Geldart et al., 2006). No caso dos pós testados, o aumento de $d_{S}$ não afetou os valores de AR e nem os de $\mathrm{IH}$, visto que os valores foram iguais se considerados os desvios-padrão das medidas. $\mathrm{O}$ $\mathrm{AR}$ de $25^{\circ}$ é característico de um material pouco coesivo, com escoamento classificado como "muito livre" (Woodcock and Mason, 1987). Os pós com IH inferiores a 1,25 também são classificados como sendo de "escoamento fácil" (Hayes, 1987).

O comportamento da densidade bulk dos pós em função do número de batidas $(\mathrm{N})$, usadas na compactação do leito, pode ser visto na Figura 3. Para N=0 determina-se a densidade bulk aerada dos pós e quando o valor de densidade se torna constante $(\mathrm{N} \rightarrow \infty)$, obtém-se a densidade bulk consolidada. Nota-se que a variação da densidade ao longo da compactação é similar para os dois pós, com um aumento inicial acentuado da densidade até $\mathrm{N}$ 50. A partir desta condição, a densidade bulk passa a aumentar lentamente em função do 
número de batidas, até atingir valores constantes. Segundo Mallol et al. (2008), no início a compactação é devida à redução do volume de vazios ou de poros grandes (muito maiores do que o tamanho médio das partículas) a volumes com tamanhos menores, da mesma ordem de grandeza do tamanho das partículas. Em seguida, tem-se o rearranjo das partículas do pó para posições mais favoráveis nos vazios do leito, diminuindo o volume total ocupado pelo pó. O valor constante foi atingido para $\mathrm{N}$ aproximadamente igual a 450 e 550, para as aluminas ALR180 e ALR100, respectivamente.

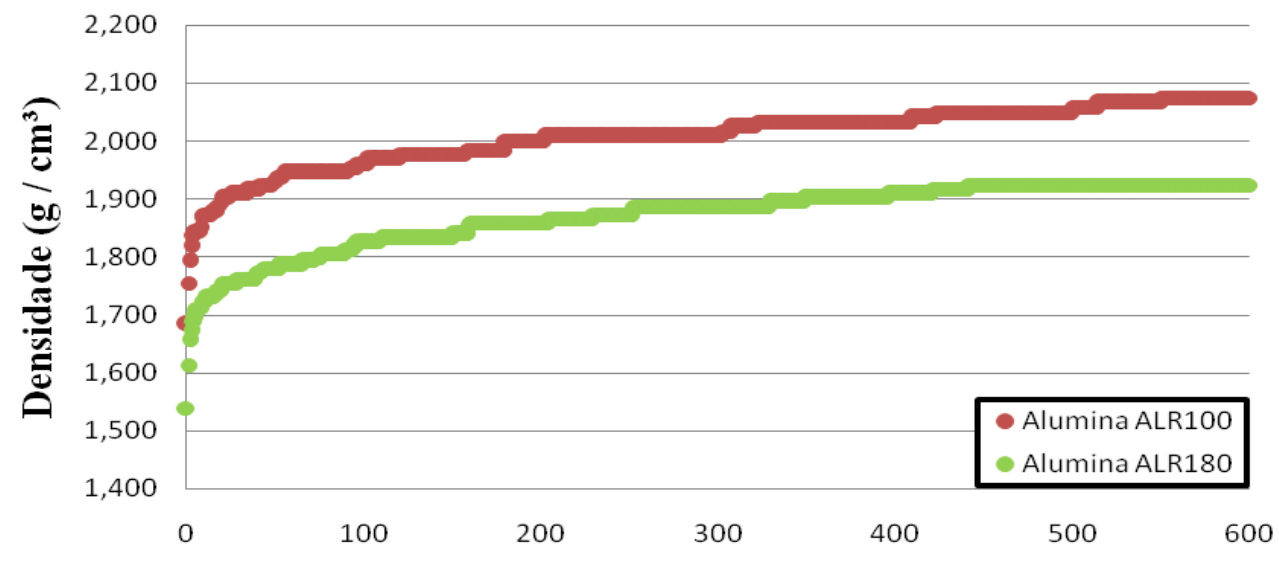

Número de batidas

Figura 3 - Cinética de compactação dos materiais particulados.

Nota-se ainda na Figura 4 que as densidades bulk da alumina ALR100 são maiores do que as da alumina ALR180. Devido à maior fração de finos da alumina ALR180 em relação a ALR100 (Figura 1), seria esperado em uma análise inicial que a densidade bulk desta fração fosse mais alta. Mas, segundo Mallol et al (2008), para pós de um mesmo material, particularmente na faixa de tamanhos menores que $200 \mu \mathrm{m}$, a densidade bulk tende a aumentar com o aumento do diâmetro de Sauter porque as forças de adesão interpartículas tendem a ser predominantes em relação à força gravitacional.

As vazões de descarga (W), medidas em triplicata foram obtidas a partir das tangentes das curvas de massa em função do tempo, como as mostradas na Figura 4. Os dados foram obtidos em triplicata e os valores médios, com os respectivos desvios-padrão, são mostrados na Tabela 2 para as diferentes condições investigadas.

Nota-se que $\mathrm{W}$ variou de acordo com o conjunto de fatores estabelecidos, entre os valores de 0,061 até $0,172 \mathrm{~g} / \mathrm{s}$. Na maioria das condições, o esvaziamento ocorreu de forma contínua e sem interrupções, corroborando o comportamento não coesivo dos pós. Na descarga das partículas mais finas no reservatório com $\alpha=45^{\circ}$ foi observado que houve uma massa de pó remanescente na secção cônica (isto ocorreu para os dois diâmetros de orifícios de saída, experimentos 2 e 4). Esta massa foi, em média, 14\% da massa total de pós alimentada, no caso do maior diâmetro de orifício, e 3\% do total, no caso do orifício de menor diâmetro. Este comportamento é um indicativo de uma mudança na vazão de 
descarga quando o nível de material no reservatório fica muito baixo (Verghese e Nedderman, 1995).

As maiores vazões foram observadas para a fração de maior diâmetro médio, escoando no orifício de maior diâmetro, enquanto as menores foram observadas na descarga da fração mais fina, escoando no orifício de menor diâmetro. Na descarga gravitacional, o aumento do diâmetro médio e da área de escoamento são fatores que afetam positivamente a vazão. A menor densidade bulk da fração mais fina (Figura 4) é um fator adicional que contribui para a redução da vazão de descarga.

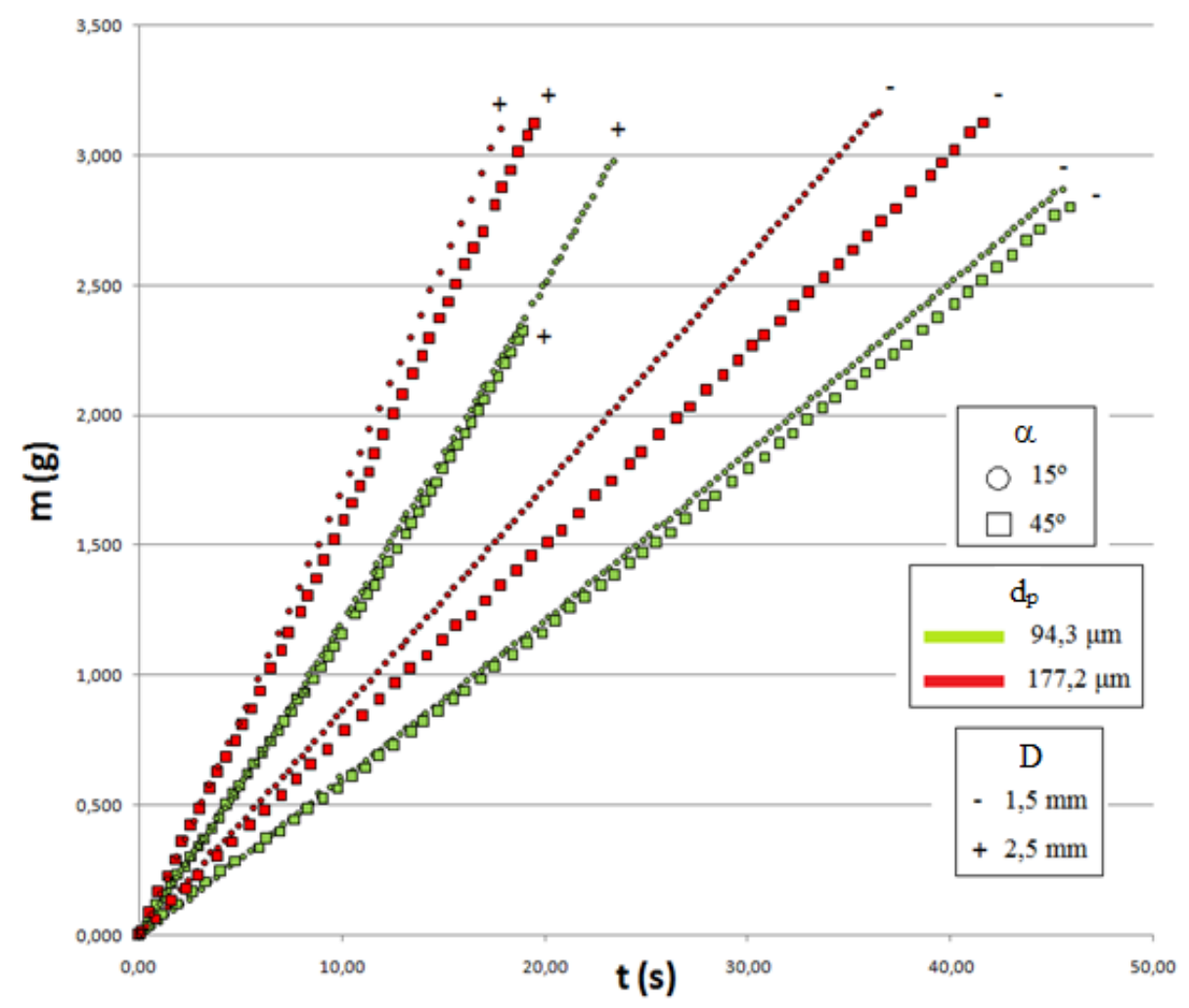

Figura 4 - Massa em função do tempo obtidos na descarga gravitacional.

A Equação (1), com a modificação proposta por Verghese e Nedderman (1995) (Equação (2)) foi utilizada para a estimativa das vazões de descarga para as condições testadas neste trabalho. Observa-se que densidade bulk recomendada para uso na correlação é a densidade bulk nas condições de escoamento, a qual é uma propriedade de difícil determinação, de acordo com os próprios autores. Assim, eles utilizaram a densidade bulk aerada, determinada experimentalmente em um consolidômetro, com a ressalva de que ela pode não representar a densidade nas condições do escoamento. Várias definições de diâmetro médio foram citadas, mas não fica claro no artigo qual foi utilizada na correlação. Adotou-se aqui o diâmetro médio de peneira $\left(\mathrm{d}_{50}\right)$. As estimativas estão apresentados na Tabela 2. Observa-se que a correlação fornece estimativas muito superestimadas das vazões de descarga, não sendo adequada para as condições testadas. Note-se que a equação foi ajustada originalmente para condições bem diferentes das deste trabalho: descarga de partículas de areia, com diâmetros 
médios entre 150 e $1540 \mu \mathrm{m}$, em reservatórios cônico-cilíndricos com $23 \mathrm{~cm}$ de diâmetro e $\alpha$ entre 10 e $60^{\circ}$.

Por meio de uma análise de variância aplicada ao planejamento experimental $2^{3}$, analisou-se a influência de $\mathrm{D}, \mathrm{d}_{\mathrm{p}}$ e $\alpha$, sobre a vazão mássica de descarga. Na Figura 5, são apresentados os gráficos comparativos para os efeitos analisados e o gráfico de Pareto, o qual ilustra os efeitos estatisticamente significativos.

Tabela 2 - Valores experimentais da vazão de descarga e valores preditos pela correlação de Verghese e Nedderman (1995)

\begin{tabular}{|c|c|c|c|}
\hline Experimento & $\begin{array}{c}\mathrm{W}_{\exp } \\
(\mathrm{g} / \mathrm{s})\end{array}$ & $\begin{array}{c}\mathrm{W}_{\text {pred }} \\
(\mathrm{g} / \mathrm{s})\end{array}$ & Desvio (\%) \\
\hline 1 & $0,063 \pm 0,002$ & 0,164 & 160 \\
\hline 2 & $0,123 \pm 0,002$ & 0,650 & 428 \\
\hline 3 & $0,127 \pm 0,002$ & 0,650 & 412 \\
\hline 4 & $0,061 \pm 0,001$ & 0,164 & 169 \\
\hline 5 & $0,159 \pm 0,002$ & 0,620 & 290 \\
\hline 6 & $0,087 \pm 0,001$ & 0,163 & 87 \\
\hline 7 & $0,075 \pm 0,001$ & 0,141 & 88 \\
\hline 8 & $0,172 \pm 0,003$ & 0,719 & 318 \\
\hline
\end{tabular}

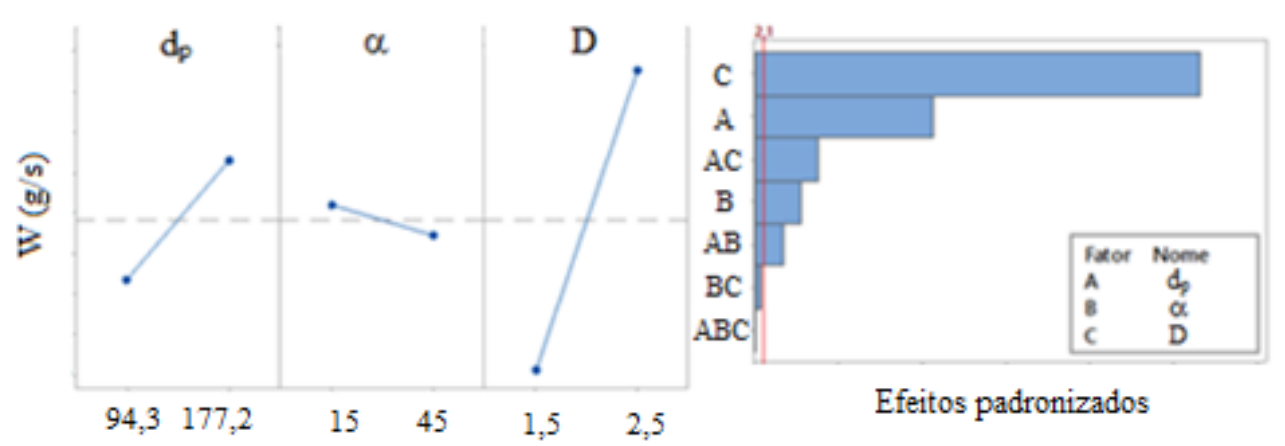

Figura 5 - Análise de variância: a) efeitos principais b) gráfico de Pareto.

Pela Figura 5-a, percebe-se que o aumento no diâmetro de saída do silo e no tamanho do material particulado acarreta um aumento da vazão de descarga, assim como o aumento da inclinação da base cônica (o menor $\alpha$ corresponde a uma parede mais inclinada), sendo que o efeito deste fator é menos importante do que os demais. Os dois valores de $\alpha$ utilizados permitem que o pó seja acomodado no reservatório com ângulos superiores ao de repouso (AR), logo as duas condições favorecem o escoamento. Observa-se no gráfico de Pareto (Figura 5-b) que todos os fatores principais tiveram efeito significativo nas vazões de descarga, sendo que os mais expressivos foram o diâmetro do orifício de descarga e o diâmetro do pó. $\mathrm{O}$ efeito da interação entre estes dois fatores foi superior ao efeito isolado do ângulo do reservatório. Efetuou-se um ajuste empírico com os 2 fatores mais 
significativos e sua interação, tendo sido obtido um bom coeficiente de correlação $\left(\mathrm{R}^{2}=\right.$ 98,63\%). A equação ajustada é dada por:

$$
W=-1,92 \times 10^{-2}-1,49 \times 10^{-4} d_{p}+3,97 \times 10^{-2} D+2,52 \times 10^{-4} D d_{p}
$$

\section{CONCLUSÕES}

Foram avaliadas as vazões mássicas na descarga gravitacional de pós de alumina com duas diferentes frações granulométricas a partir de reservatórios cônico-cilíndricos. Foi constatado que as vazões de descarga aumentam com o aumento do tamanho médio das partículas e com o aumento do diâmetro do orifício de descarga. Dentre os fatores avaliados, o ângulo de inclinação do reservatório foi o fator com menor influência na vazão de descarga. A correlação empírica proposta por Veghese e Nedderman (1995) superestima as vazões de descarga, mas foi possível ajustar estatisticamente uma equação para estimar as vazões de descarga nas condições avaliadas.

\section{AGRADECIMENTOS}

À FAPESP pela bolsa de IC e ao Centro de Secagem/DEQ pelo espaço físico.

\section{REFERÊNCIAS}

CAMPOS, M.M., FERREIRA, M.C.. A comparative analysis of the flow properties between two alumina-based powders. Advances in Material Science and Engineering, article ID 519846, 7 pages, 2013.

GELDART, D. et al. Characterization of powder flowability using measurement of angle of repose. China Particuology, vol. 4, p. 104-107, 2006.

GOMIDE, R. Operações Unitárias: operações com sistemas sólidos granulares. Edição do Autor, Vol. 1, São Paulo, 1983.

HAYES, G. D. Food Engineering Data Handbook. New York: Wiley, p.83 1987.

MALLOL, G.; AMORÓS, J. L.; ORTS, M. J.; LLORENS, D. Densification of monomodal quartz particle beds by tapping. Chemical Engineering Science, vol. 63, p. 5447-5456, 2008 .

VERGHESE, T.M.; NEDDERMAN, R.M. The discharge of fine sands from conical hoppers. Chemical Engineering Science, vol. 50, p. 3143-3153, 1995.

WOODCOCK, C.R.; MASON, J.S. Bulk Solids Handling - An Introduction to the Practice and Technology. Glasgow: Leonard Hill, 1987. 